\title{
Synaptic Properties of the Mammillary and Cortical Afferents to the Anterodorsal Thalamic Nucleus in the Mouse
}

\author{
Iraklis Petrof and S. Murray Sherman \\ Department of Neurobiology, University of Chicago, Chicago, Illinois 60637
}

\begin{abstract}
Input to sensory thalamic nuclei can be classified as either driver or modulator, based on whether or not the information conveyed determines basic postsynaptic receptive field properties. Here we demonstrate that this distinction can also be applied to inputs received by nonsensory thalamic areas. Using flavoprotein autofluorescence imaging, we developed two slice preparations that contain the afferents to the anterodorsal thalamic nucleus (AD) from the lateral mammillary body and the cortical afferents arriving through the internal capsule, respectively. We examined the synaptic properties of these inputs and found that the mammillothalamic pathway exhibits paired-pulse depression, lack of a metabotropic glutamate component, and an all-or-none response pattern, which are all signatures of a driver pathway. On the other hand, the cortical input exhibits graded paired-pulse facilitation and the capacity to activate metabotropic glutamatergic responses, all features of a modulatory pathway. Our results extend the notion of driving and modulating inputs to the $\mathrm{AD}$, indicating that it is a first-order relay nucleus and suggesting that these criteria may be general to the whole of thalamus.
\end{abstract}

\section{Introduction}

The thalamus receives input from a multitude of cortical and subcortical areas and relays this to cortex, representing the sole source of information flow to the latter. Afferents to the main sensory thalamic nuclei can be divided into drivers, which determine the main postsynaptic receptive field properties and carry the main information to be relayed to cortex, and modulators, which affect the way driver information is relayed (Sherman and Guillery, 1998, 2006). Driver inputs exhibit such synaptic features as a strong, all-or-none response pattern, paired-pulse depression, and the capacity to activate only ionotropic glutamate receptors. Modulators, on the other hand, produce relatively weak, graded responses with paired-pulse facilitation and can activate both ionotropic and metabotropic receptors (Sherman and Guillery, 2006). Individual thalamic nuclei, on the basis of whether they relay driving information from subcortical areas to the cortex or from one cortical area to another, have been categorized as first-order (FO) or higher-order (HO) nuclei, respectively (Guillery, 1995; Sherman and Guillery, 1998, 2006).

Whereas a great deal is known about the origin of driver and modulating input in most traditional sensory thalamic nuclei (Guillery et al., 2001; Wang et al., 2002; Li et al., 2003; Reichova

Received April 1, 2009; revised May 7, 2009; accepted May 22, 2009.

Funding was obtained from National Institutes of Health (NIH)/National Eye Institute Grant EY03038 and NIH/ National Institute on Deafness and Other Communication Disorders Grant DC008794 to S.M.S. We thank the following people for their invaluable help and technical advice in carrying out all experimental procedures (in alphabetical order): Dr. Joseph A. Beatty, Elise N. Covic, Dr. Y. W. Lam, Dr. Charles C. Lee, Dr. Dan A. Llano, Brian B. Theyel, and Dr. Carmen Varela.

Correspondence should be addressed to S. Murray Sherman, Department of Neurobiology, University of Chicago, Abbott J-117, 947 East 58th Street, Chicago, IL 60637. E-mail: msherman@bsd.uchicago.edu.

D0I:10.1523/JNEUROSCI.1564-09.2009

Copyright $\odot 2009$ Society for Neuroscience $\quad$ 0270-6474/09/297815-05\$15.00/0 and Sherman, 2004; Van Horn and Sherman, 2007; Lee and Sherman, 2008), this is not the case for other nuclei. It needs to be noted that the distinction between "traditional sensory" and "other" thalamic nuclei is primarily a semantic one. By "traditional sensory nuclei," we mean nuclei such as the lateral geniculate or ventral posterior medial nuclei, which have been the main experimental models to study thalamus. One of the "other" nuclei is the anterodorsal nucleus (AD), perhaps the most prominent of the anterior thalamic nuclei. Its subcortical afferents arrive from lateral mammillary body (LM) of the hypothalamus (Watanabe and Kawana, 1980; Hayakawa and Zyo, 1989; Shibata, 1992), whereas cortical afferents arrive primarily from the parasubiculum, postsubiculum, and the parts of the retrosplenial cortex (van Groen and Wyss, 1990a,b; Shibata and Naito, 2005), the latter two also being the major target of $\mathrm{AD}$ efferents (Shibata, 1993; Van Groen and Wyss, 1995, 2003). In addition to their involvement in some limbic functions (Sziklas and Petrides, 1999; Célérier et al., 2000; Wolff et al., 2008), AD cells have been found to respond to head direction activity, similarly to cells in cortical areas associated with AD (Sharp, 1996; Goodridge and Taube, 1997; Hargreaves et al., 2007). We wished to determine whether the synaptic properties of various inputs to AD conformed to the driver/modulator model developed for the traditional sensory thalamic relays, thereby extending this concept to thalamus more generally, or whether some other pattern might exist. To do so, we developed two new slice preparations in the mouse: one that contains the pathway between LM and AD, and another that contains the severed corticothalamic axons terminating into $\mathrm{AD}$ through the internal capsule. We thus examined the synaptic responses of $\mathrm{AD}$ cells following the stimulation of their subcortical and cortical afferent pathways. Our data show that the mammillary input to $\mathrm{AD}$ possesses the profile character- 


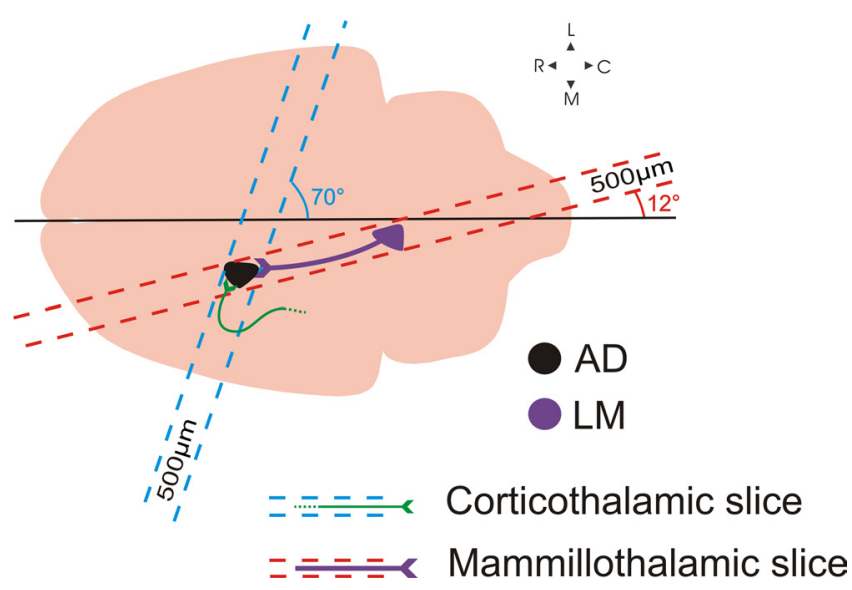

Figure 1. Schematic illustration of the blocking angles used to generate the two slice preparations. The mammillothalamic slice was prepared as follows: taking the midline as a reference, a $12^{\circ}$ cut was made to parallel the route of the mammillothalamic tract and a single $500-\mu \mathrm{m}$-thick slice was vibratomed. The slice containing the severed cortical axons into AD was prepared by blocking the brain at a $70^{\circ}$ angle and by cutting a single $500-\mu \mathrm{m}$-thick slice. $\mathrm{C}$, Caudal; L, lateral; $M$, medial; R, rostral.

istics of a driving pathway, whereas cortical input arriving through the internal capsule appears to be of an exclusively modulatory nature, a pattern seen in the traditional sensory thalamic nuclei.

\section{Materials and Methods}

Slice preparation. BALB/c mice (ages $14-18 \mathrm{~d}$ ) were anesthetized with isoflurane and decapitated. Brains were rapidly removed and placed in chilled $\left(0-4^{\circ} \mathrm{C}\right)$, oxygenated $\left(95 \% \mathrm{O}_{2}, 5 \% \mathrm{CO}_{2}\right)$ slicing solution containing the following (in mM): $2.5 \mathrm{KCl}, 1.25 \mathrm{NaH}_{2} \mathrm{PO}_{4}, 10 \mathrm{MgCl}_{2}, 0.5 \mathrm{CaCl}_{2}$, $26 \mathrm{NaHCO}_{3}, 11$ glucose, and 206 sucrose. The mammillothalamic slice preparation was prepared as follows: brains were blocked at a $12^{\circ}$ angle from the midline over the right hemisphere (Fig. 1), and the blocked side was glued onto a vibratome platform (Leica). Slices were cut at $500 \mu \mathrm{m}$ off-parasagittal in that orientation, thus preserving most of the connectivity between $\mathrm{LM}$ and $\mathrm{AD}$ (see Results). The trajectory of the corticofugal fibers terminating in $\mathrm{AD}$ is such that the inclusion of the whole pathway in a single slice was not possible. We therefore had to develop a slice that would contain as much of the corticothalamic axons as possible to allow us to maximize the distance between the stimulation site and the imaged/recorded site in $\mathrm{AD}$. For the corticothalamic slice preparation, brains were blocked at a $70^{\circ}$ off-coronal angle (Fig. 1), the blocked area was glued to the vibratome platform, and slices were cut at $500 \mu \mathrm{m}$ in that orientation. Once cut, slices from either preparation were placed in warm $\left(32^{\circ} \mathrm{C}\right.$ ) oxygenated artificial CSF (ACSF) [containing (in mM): $125 \mathrm{NaCl}$, $3 \mathrm{KCl}, 1.25 \mathrm{NaH}_{2} \mathrm{PO}_{4}, 1 \mathrm{MgCl}_{2}, 2 \mathrm{CaCl}_{2}, 25 \mathrm{NaHCO}_{3}$, and 25 glucose] for $30 \mathrm{~min}$ and were then allowed to recover for at least an additional $30 \mathrm{~min}$, at room temperature, before being used. Once in the recording chamber, slices were continuously perfused with oxygenated ACSF.

Flavoprotein autofluorescence imaging. The viability of connectivity in the two slice preparations was assessed using imaging of flavoprotein autofluorescence (FA), details of which have been described by Llano et al. (2009). Briefly, FA captures green light $(520-560 \mathrm{~nm})$ emitted by mitochondrial flavoproteins in the presence of blue light $(450-490 \mathrm{~nm})$ in conditions of cellular metabolic activity (see Shibuki et al., 2003). This method allows the detection of cellular activation (through the elevated levels of mitochondrial green light emissions) once a connected presynaptic area has been stimulated. FA was performed on a fluorescent lightequipped microscope (Axioscop 2FS, Carl Zeiss Instruments) connected with a QImage Retiga-SRV camera (QImaging Corporation).

For the assessment of the mammillothalamic and corticothalamic slices, LM and the dorsal internal capsule were respectively stimulated using a bipolar concentric electrode (FHC) while simultaneously imaging the postsynaptic activation in the anterior thalamus. Electrical stim- ulation consisted of a current injection train $(50-250 \mu \mathrm{A}, 20 \mathrm{~Hz})$ over $1 \mathrm{~s}$. FA activity was monitored over that period of time in addition to $1 \mathrm{~s}$ before stimulation and $12 \mathrm{~s}$ following stimulation. Fluorescence images were acquired at 2.5-10 frames per second (integration time of 150-300 $\mathrm{ms})$. The optical image was generated as a function of the $\Delta \mathrm{f} / \mathrm{f}$ ratio of the baseline autofluorescence of the slice before stimulation subtracted from the autofluorescence of the slice over the period of stimulation $(\Delta f)$ divided by baseline $(f)$.

In some FA experiments, LM was stimulated through the photouncaging of caged glutamate. For these experiments, nitroindolinylcaged glutamate (Sigma-Aldrich) was added to the recirculating ACSF (0.4 $\mathrm{mM}$ ), and a UV laser beam (DPSS Laser) was used to locally photolyse the caged compound (Shepherd et al., 2003; Lam and Sherman, 2005, 2007; Lam et al., 2006). The laser beam had an intensity of 20-80 $\mathrm{mW}$ and gave off a $10 \mathrm{~ms}, 20$-pulse light stimulus (355 nm wavelength, frequency-tripled Nd:YVO4, $100 \mathrm{kHz}$ pulse repetition rate). For photouncaging FA experiments, the $\Delta f / f$ ratio was calculated by subtracting baseline autofluorescence from autofluorescence immediately after the last laser pulse $(\Delta f)$ and by then dividing the result by baseline $(f)$. FA analyses for both electrical and glutamate photo-uncaging stimulation were performed using a custom-made software written in Matlab (MathWorks).

Electrophysiology. Whole-cell patch-clamp recordings in currentclamp mode were performed using a visualized slice setup under a differential interference contrast-equipped Axioscop 2FS microscope (Carl Zeiss Instruments) and with a Multiclamp 700B amplifier and pCLAMP software (Molecular Devices). The pipettes used for the recordings were pulled from $1.5 \mathrm{~mm}$ borosilicate glass capillaries and were filled with intracellular solution containing the following (in mM): $117 \mathrm{~K}$-gluconate, $13 \mathrm{KCl}, 1 \mathrm{MgCl}_{2}, 0.07 \mathrm{CaCl}_{2}, 10$ HEPES, 0.1 EGTA, $2 \mathrm{Na}_{2}$-ATP, and 0.4 Na-GTP, pH 7.3, 290 mOsm). Pipette input resistances varied between 4 and $8 \mathrm{M} \Omega$. Data were digitized on a Digidata 1200 board and stored on a computer. Analyses of the acquired traces were performed in ClampFit (Molecular Devices) software. To evoke synaptic responses in AD cells, electric stimulation of their afferent pathways was delivered through the same bipolar electrodes as the ones used for FA. For the examination of short-term synaptic plasticity, we used a protocol that consisted of four 0.1-ms-long pulses at a frequency of $10 \mathrm{~Hz}$. In doing so, we used the minimal stimulation intensity required to elicit an EPSP. This was done to minimize the potential spread of current around the stimulation area, which could potentially result in the recruitment of other $\mathrm{AD}$ afferents, thus contaminating the responses recorded from the nucleus. Progressively higher intensities were used to test for a graded or an all-or-none response profile (see Results). The stimulation intensities that were used typically varied between 10 and $350 \mu \mathrm{A}$. For the examination of metabotropic glutamate responses, we used a high-frequency stimulation (HFS) protocol that delivered 0.1 -ms-long pulses at $125 \mathrm{~Hz}$ over $800 \mathrm{~ms}$.

All experimental protocols (including FA ones) were performed in the presence of $\mathrm{GABA}_{\mathrm{A}}$ and $\mathrm{GABA}_{\mathrm{B}}$ receptor antagonists (SR95531 [2-(3carboxyl)-3-amino-6-(4-methoxyphenyl)-pyridazinium bromide], 50 nM, and CGP46381 [(3-aminopropyl)(cyclohexylmethyl)phosphinic acid], $50 \mathrm{~nm}$, respectively). In addition, to isolate any metabotropic responses that might be present, HFS was applied in the presence of NMDA and AMPA antagonists [5H-dibenzo[ $a, d]$ cyclohepten-5,10-imine (MK$801), 40 \mu \mathrm{M}$, and DNQX, $50 \mu \mathrm{M}$, respectively]. Finally, the metabotropic glutamate receptor 1 (mGluR1) receptor antagonist LY367385 (40 $\mu \mathrm{M})$ was used to block metabotropic responses, when present.

\section{Results}

\section{Viability of slice connectivity}

Figure 2, $A$ and $B$, shows the FA images during electrical stimulation of LM and the corticothalamic axons terminating in $\mathrm{AD}$, respectively. In both cases, an activation can be seen in $\mathrm{AD}$ remote from the stimulation site. An activation of $\mathrm{AD}$ was also seen when the stimulation of LM was performed through the photouncaging of caged glutamate (Fig. $2 E$ ). We thus conclude that the imaged $\mathrm{AD}$ activation was the direct outcome of mammillary 
A Stimulation of Mammillary Bodies
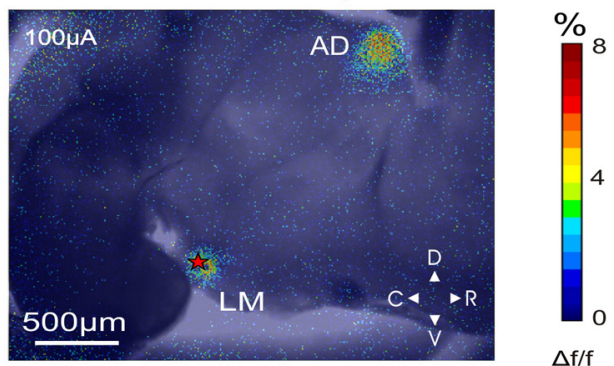

C Paired-Pulse Depression All-or-None Response (Mammillothalamic)

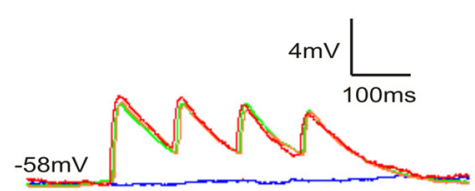

$\mathrm{Ca}$

$-57 \mathrm{mV} \quad 250 \mu \mathrm{A}$

$\mathrm{DNQX}+\mathrm{MK}-801$

E Photo-stimulation of LM

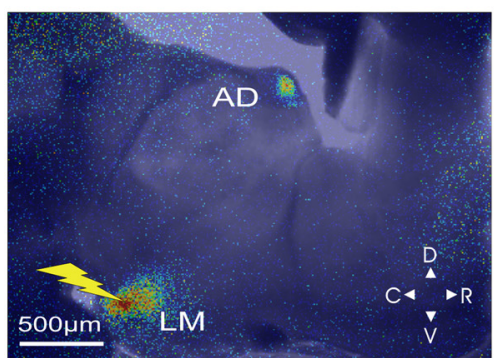

B Stimulation of Corticothalamic Axons

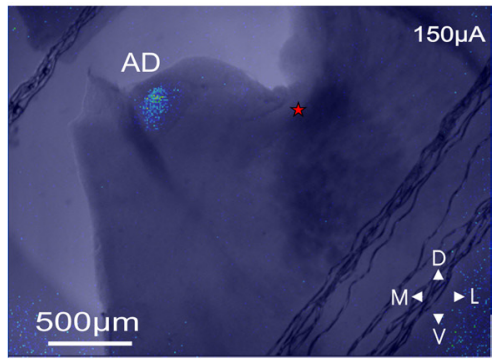

D

Paired-Pulse Facilitation Graded Response (Corticothalamic)

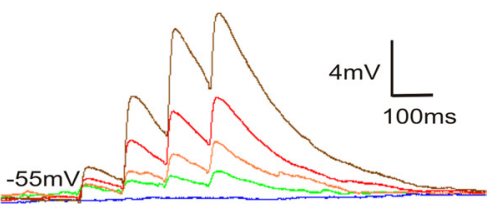

\section{$\mathrm{Da}$}

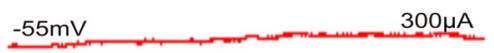

DNQX + MK-801

F

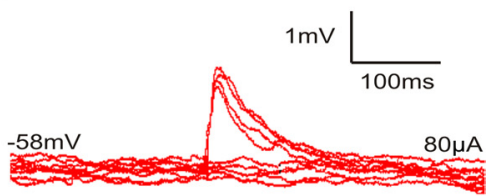

HFS: Metabotropic Receptor activation

(Mammillothalamic)

G

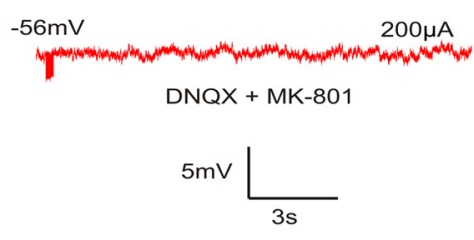

(Corticothalamic)

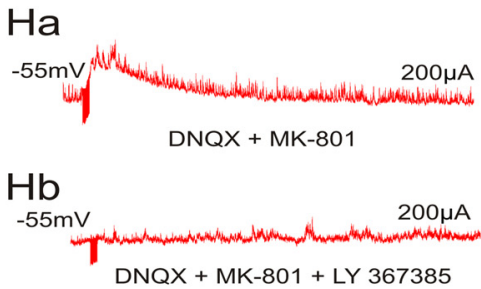

ACPD application (High $\mathrm{Mg}^{2+} /$ low $\mathrm{Ca}^{2+}$ ACSF)

I

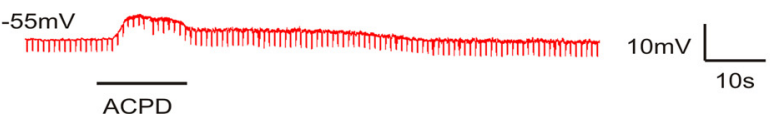

Figure 2. $\quad A, B$, Examples of superimposed bright-field and AF images illustrating the areas of activation during the stimulation of the mammillary bodies and the corticothalamic axons of $A D$, respectively (red stars indicate the area of stimulation). Stimulation in $\boldsymbol{A}$ resulted in paired-pulse depression ( $\boldsymbol{C}$, whereas stimulation in $\boldsymbol{B}$ resulted in paired-pulse facilitation (D). The color panel represents the percentage of $F A$ signal change from prestimulation baseline. $C$, Demonstration of the all-or-none response profile in $A D$ following mammillary stimulation of increasing intensity. $D$, Demonstration of the graded response profile in $A D$ following corticothalamic axon stimulation of increasing intensity. $\mathbf{C a}, \mathbf{D a}$, Responses in both types of synapses were blocked when MK-801 and DNQX (NMDA and AMPA antagonist, respectively) were applied to the bath. All traces represent the average of 10 sweeps. $\boldsymbol{E}_{\text {, }}$ Example of a superimposed AF and bright-field image illustrating the areas of activation during glutamate photo-uncaging

stimulation and not the product of any activated axons of passage.

\section{Driver versus modulator}

response signatures

We recorded from $32 \mathrm{AD}$ cells with resting membrane potentials of $-53.1 \pm 5.1 \mathrm{mV}$ (uncorrected for an approximately -10 $\mathrm{mV}$ gap potential) and input resistances of $235.6 \pm 157.8 \mathrm{M} \Omega$. For the $16 \mathrm{AD}$ cells recorded in the mammillary slice, lowfrequency $(10-20 \mathrm{~Hz})$ stimulation of LM that exceeded $50 \mu \mathrm{A}$ evoked EPSPs in the recorded AD cells. These EPSPs showed paired-pulse depression (mean EPSP2/ EPSP1 ratio: $0.73 \pm 0.1$ ) (Figs. $2 C, 3$, bottom) and an all-or-none activation pattern (Figs. 2C, 3, top, a). That is, in order for EPSPs to be produced, a certain threshold of stimulation intensity had to be reached, after which further increases in stimulation intensity did not significantly increase the amplitude of the EPSPs. These EPSPs could be completely blocked by introducing ionotropic receptor (AMPA and NMDA) antagonists (DNQX, $50 \mu \mathrm{M}$, and MK-801, $40 \mu \mathrm{M}$, respectively) in the bath (Fig. $2 \mathrm{Ca}$ ). In the presence of these antagonists, HFS $(120 \mathrm{~Hz})$ did not produce any changes in cell membrane potential, indicating a lack of involvement of metabotropic receptors evoked by the mammillothalamic synapse (Fig. 2G).

The synaptic properties revealed by stimulation of the dorsal internal capsule stimulation in 16 other $\mathrm{AD}$ cells in the other slice configuration were dramatically different from those of LM. The EPSPs produced from such stimulation showed paired-pulse facilitation (mean EPSP2/EPSP1 ratio: $2.53 \pm 1.34$ ) (Figs. $2 D, 3$, top, $b$, and bottom), and they showed a graded activation pattern, meaning that their amplitude demonstrated a positive correlation with the intensity of the injected current (Figs. 2D, 3, top, $b$ ). The evoked EPSPs generated by lowfrequency activation of the cortical axons

(yellow bolt) over the mammillary bodies. The activation color scheme is the same as in $\boldsymbol{A}$ and $\boldsymbol{B}$. $\boldsymbol{F}$, Minimal stimulation intensity for depressing synapses was defined as the intensity that resulted in 50\% failures in producing an EPSP. G, HFS $(125 \mathrm{~Hz})$ of $L M$ in the presence of ionotropic receptor antagonists resulted in no changes in the membrane potential of AD cells. $\boldsymbol{H}$, Stimulation of the corticothalamic axons under these conditions resulted in a slow and prolonged membrane depolarization of $A D$ cells $(\boldsymbol{a})$ that could be blocked with LY367385 (mGluR1 antagonist) (b). I, Membrane depolarization seen after the application of ACPD in the slice in the presence of high- $\mathrm{Mg}^{2+} /$ low-Ca ${ }^{2+}$ ACSF. C, Caudal; D, dorsal; L, lateral; $M$, medial; $R$, rostral; $V$, ventral. 

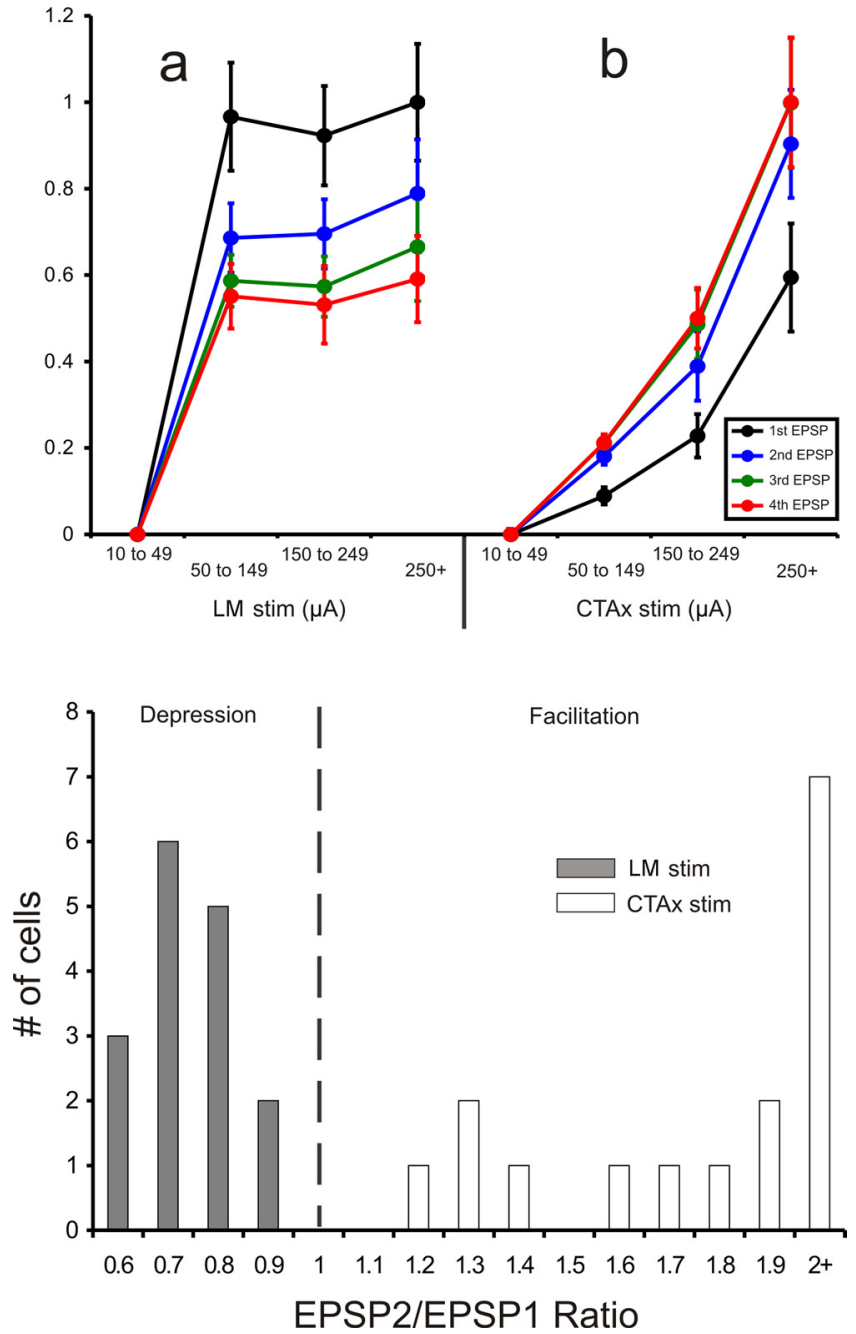

Figure 3. Top, Normalized EPSP amplitudes recorded in AD following the stimulation of $L M$ $(\boldsymbol{a})$ and the corticothalamic axons $(\boldsymbol{b})$ at different intensities. The data are plotted as a function of each EPSP (first to fourth) for each intensity bin. Stimulation of LM $(n=16)$ resulted in an all-or-none response pattern: once the current intensity threshold for the generation of EPSPs is reached, further increases in current intensity do not produce larger EPSPs. Contrary to that, increases in the intensity of stimulation of the corticothalamic axons $(n=16)$ resulted in increases in the amplitude of the EPSPs recorded in AD. Error bars represent the SEM. Bottom, Distribution of the paired-pulse effects in the two pathways plotted as the ratio of the amplitude of the second EPSP divided by the amplitude of the first EPSP, as generated at minimal stimulation intensity. The mammillothalamic synapse produced ratios of $<1$ (depression), while the corticothalamic synapse produced ratios of $>1$ (facilitation). The EPSP2/EPSP1 ratios of the two populations were significantly different ( $p<0.001, t$ test). CTAx, Corticothalamic axons.

were blocked by ionotropic receptor antagonists (Fig. 2Da). However, in the presence of these antagonists, HFS produced a prolonged depolarization ( $9 \mathrm{~s}, 4 \mathrm{mV}$ ) of the cells' membrane potential (Fig. $2 \mathrm{Ha}$ ). This depolarization could be blocked by the mGluR1 antagonist LY367385 (Fig. 2 Hb). To examine whether the metabotropic responses could be postsynaptic (i.e., the result of metabotropic receptors in the recorded $\mathrm{AD}$ cell) and not presynaptic (i.e., the result of presynaptic metabotropic receptors on the corticothalamic axons and/or terminals), we applied the general mGluR agonist $1 S, 3 R-1$ amino-1,3-cyclopentanedicarboxylate (ACPD) (final concentration: $65 \mu \mathrm{M})$ in the presence of high $-\mathrm{Mg}^{2+} /$ low- $-\mathrm{Ca}^{2+}$ ACSF, which blocks synaptic transmission. This control was performed on five cells, three in the mammillothalamic and two in the corticothalamic preparation. The membrane depo- larization for all five cells produced by ACPD in these conditions (Fig. 2I) demonstrated that the metabotropic effect was postsynaptic to the recorded cell (mean depolarization for $\mathrm{AD}$ cells sampled in the mammillothalamic slice: $11.6 \pm 1.5 \mathrm{mV}$, and in the corticothalamic slice: $10 \pm 2.8 \mathrm{mV}$ ).

\section{Discussion}

The examination of the synaptic properties of the mammillothalamic and corticothalamic afferents of $\mathrm{AD}$ revealed that the two pathways possess different functional profiles. The mammillothalamic input to $\mathrm{AD}$ was found to possess driver characteristics such as a lack of metabotropic receptor activation and large, allor-none EPSPs that manifest synaptic depression. These synaptic features reflect a high probability of transmitter release and a fast postsynaptic relay of information (Sherman and Guillery, 2006). On the other hand, the generation of graded, facilitating EPSPs and the activation of metabotropic receptors on AD cells following corticothalamic stimulation reflect a longer, yet overall weaker, influence onto these cells. Such an influence most likely operates in modulating the way driving input is relayed (Sherman and Guillery, 2006).

The pattern of cortical and subcortical synaptic relationships of $\mathrm{AD}$, therefore, resembles that of the sensory FO thalamic nuclei, such as the lateral geniculate, the ventral posterior, and the ventral medial geniculate nuclei (Reichova and Sherman, 2004; Sherman and Guillery, 2006; Lee and Sherman, 2008). These nuclei receive their driving input from subcortical centers, whereas their cortical input arrives through axons of cortical cells in layer 6 and possesses modulatory synaptic characteristics. Although $\mathrm{HO}$ sensory thalamic nuclei also receive modulatory input from cortical layer 6 , they receive driving input that arises from cortical layer 5 (Guillery, 1995; Reichova and Sherman, 2004; Sherman and Guillery, 2006). In the present study, we stimulated the whole extent of the severed cortical axons reaching $\mathrm{AD}$ through the internal capsule, thus activating axons potentially originating in either of these layers. The absence of driver-like responses leads us to the conclusion that the cortical input to AD arises exclusively from layer 6 , which agrees with previous anatomical reports from the rat (van Groen and Wyss, 1990a).

However, not all cortical afferents of $\mathrm{AD}$ arrive through the traditional internal capsular route. Axons of cortical cells also reach AD through the fornix (Shibata, 1998), a fiber tract that carries cortical and hippocampal input into areas of the hypothalamus, including the mammillary bodies (Swanson and Cowan, 1977; Donovan and Wyss, 1983; Shibata, 1989). This input uses an unusual route to reach the thalamus, namely via an anterior and medial trajectory, thus apparently avoiding a transit through the thalamic reticular nucleus (TRN), something that is not the case for most, if not all, other corticothalamic pathways, regardless of whether they innervate TRN or not. Because of the geometry and close proximity to $\mathrm{AD}$ of corticothalamic axons passing through the fornix, we could not isolate this corticothalamic input for stimulation. Thus while our data do not completely rule out the possibility that $\mathrm{AD}$ receives some driving input from layer 5 of cortex via the fornix route, other data do seem to do so. That is, Shibata (1998) reported that the multiple cortical areas retrogradely labeled from tracer injections into AD labeled only layer 6 neurons and not those in layer 5. Since layer 5 is the source of driver input to HO nuclei (Sherman and Guillery, 2006), and none seem to be involved in the cortical projection to $\mathrm{AD}$, we conclude that $\mathrm{AD}$ is an $\mathrm{FO}$ nucleus with its subcortical driving input from the mammillary bodies.

Cells in AD and its associated cortical areas (as well as the 
mammillary bodies) appear to fire in accordance to the organism's head direction movements (Sharp, 1996; Goodridge and Taube, 1997; Stackman and Taube, 1998; Hargreaves et al., 2007). Previous reports combining lesions with electrophysiological recordings demonstrate that head direction activity in postsubiculum is dependent on $\mathrm{AD}$ but not vice versa (Goodridge and Taube, 1997). More specifically, lesions in AD result in the abolition of head direction-related activity in the postsubiculum, whereas lesions of postsubiculum affect only secondary features of head direction-related activity in $\mathrm{AD}$, such as the range of directional firing. This suggests that driving information regarding head movement is not arriving to $\mathrm{AD}$ from cortex but that $\mathrm{AD}$ relays such information to cortex instead. This agrees with our results showing that cortical information coming into $\mathrm{AD}$ is of a modulatory nature and suggests that the driving head direction information reaching postsubiculum through $\mathrm{AD}$ may be arriving to the latter from the mammillary region.

Overall our results demonstrate that the distinction between driving and modulating inputs is not restricted exclusively to traditional sensory thalamic nuclei, but it may apply more broadly to thalamus. Understanding the functional organization of a transthalamic pathway cannot be achieved by examining the relative numerical strength of its afferents, given that these can exert significantly diverse postsynaptic effects of equally diverse functional importance. A better understanding of the above could therefore be offered through the identification of the origin of driving afferents in a thalamic area, a method the significance of which has been largely overlooked. This could represent a potentially useful tool in understanding not only the functional role of transthalamic pathways but, by extension, that of the cortical areas to which they project.

\section{References}

Célérier A, Ognard R, Decorte L, Beracochea D (2000) Deficits of spatial and non-spatial memory and of auditory fear conditioning following anterior thalamic lesions in mice: comparison with chronic alcohol consumption. Eur J Neurosci 12:2575-2584.

Donovan MK, Wyss JM (1983) Evidence for some collateralization between cortical and diencephalic efferent axons of the rat subicular cortex. Brain Res 259:181-192.

Goodridge JP, Taube JS (1997) Interaction between the postsubiculum and anterior thalamus in the generation of head direction cell activity. J Neurosci 17:9315-9330.

Guillery RW (1995) Anatomical evidence concerning the role of the thalamus in corticocortical communication: a brief review. J Anat 187:583-592.

Guillery RW, Feig SL, Van Lieshout DP (2001) Connections of higher order visual relays in the thalamus: a study of corticothalamic pathways in cats. J Comp Neurol 438:66-85.

Hargreaves EL, Yoganarasimha D, Knierim JJ (2007) Cohesiveness of spatial and directional representations recorded from neural ensembles in the anterior thalamus, parasubiculum, medial entorhinal cortex and hippocampus. Hippocampus 17:826-841.

Hayakawa T, Zyo K (1989) Retrograde double-labeling study of the mammillothalamic and the mammillotegmental projections in the rat. J Comp Neurol 284:1-11.

Lam YW, Sherman SM (2005) Mapping by laser photostimulation of connections between the thalamic reticular and ventral posterior lateral nuclei in the rat. J Neurophysiol 94:2472-2483.

Lam YW, Sherman SM (2007) Different topography of the reticulothalmic inputs to first- and higher-order somatosensory thalamic relays revealed using photostimulation. J Neurophysiol 98:2903-2909.

Lam YW, Nelson CS, Sherman SM (2006) Mapping of the functional interconnections between thalamic reticular neurons using photostimulation. J Neurophysiol 96:2593-2600.
Lee CC, Sherman SM (2008) Synaptic properties of thalamic and intracortical inputs to layer 4 of the first- and higher-order cortical areas in the auditory and somatosensory systems. J Neurophysiol 100:317-326.

Li J, Guido W, Bickford ME (2003) Two distinct types of corticothalamic EPSPs and their contribution to short-term synaptic plasticity. J Neurophysiol 90:3429-3440.

Llano DA, Theyel BB, Mallik AK, Sherman SM, Issa NP (2009) Rapid and sensitive mapping of long range connections in vitro using flavoprotein autofluorescence imaging combined with laser photostimulation. J Neurophysiol 101:3325-3340.

Reichova I, Sherman SM (2004) Somatosensory corticothalamic projections: distinguishing drivers from modulators. J Neurophysiol 92:2185-2197.

Sharp PE (1996) Multiple spatial/behavioural correlates for cells in the rat postsubiculum: multiple regression analysis and comparison to other hippocampal areas. Cereb Cortex 6:238-259.

Shepherd GM, Pologruto TA, Svoboda K (2003) Circuit analysis of experience-dependent plasticity in the developing rat barrel cortex. Neuron 38:277-289.

Sherman SM, Guillery RW (1998) On the actions that one nerve cell can have on another: distinguishing "drivers" from "modulators." Proc Natl Acad Sci U S A 95:7121-7126.

Sherman SM, Guillery RW (2006) Exploring the thalamus. Cambridge, MA: MIT.

Shibata H (1989) Descending projections to the mammillary nuclei in the rat, as studied by retrograde and anterograde transport of wheat germ agglutinin-horseradish peroxidase. J Comp Neurol 285:436-452.

Shibata H (1992) Topographic organization of subcortical projections to the anterior thalamic nuclei in the rat. J Comp Neurol 323:117-127.

Shibata H (1993) Efferent projections from the anterior thalamic nuclei to the cingulate cortex in the rat. J Comp Neurol 330:533-542.

Shibata H (1998) Organization of projections of rat retrosplenial cortex to the anterior thalamic nuclei. Eur J Neurosci 10:3210-3219.

Shibata H, Naito J (2005) Organization of anterior cingulate and frontal cortical projections to the anterior and laterodorsal thalamic nuclei in the rat. Brain Res 1059:93-103.

Shibuki K, Hishida R, Murakami H, Kudoh M, Kawaguchi T, Watanabe M, Watanabe S, Kouuchi T, Tanaka R (2003) Dynamic imaging of somatosensory cortical activity in the rat visualized by flavoprotein autofluorescence. J Physiol 549:919-927.

Stackman RW, Taube JS (1998) Firing properties of the rat lateral mammillary single units: head direction, head pitch, and angular head velocity. J Neurosci 18:9020-9037.

Swanson LW, Cowan WM (1977) An autoradiographic study of the organization of the efferent connections of the hippocampal formation in the rat. J Comp Neurol 172:49-84.

Sziklas V, Petrides M (1999) The effects of lesions to the anterior thalamic nuclei on object-place associations in rats. Eur J Neurosci 11:559-566.

van Groen T, Wyss JM (1990a) The connections of presubiculum and parasubiculum in the rat. Brain Res 518:227-243.

van Groen T, Wyss JM (1990b) The postsubicular cortex in the rat: characterization of the fourth region of the subicular cortex and its connections. Brain Res 529:165-177.

Van Groen T, Wyss JM (1995) Projections from the anterodorsal and anteroventral nucleus of the thalamus to the limbic cortex in the rat. J Comp Neurol 358:584-604

Van Groen T, Wyss JM (2003) Connections of the retrosplenial granular b cortex in the rat. J Comp Neurol 463:249-263.

Van Horn SC, Sherman SM (2007) Fewer driver synapses in higher order than in first order thalamic relays. Neuroscience 146:463-470.

Wang S, Eisenback MA, Bickford ME (2002) Relative distribution of synapses in the pulvinar nucleus of the cat: implications regarding the "driver/modulator" theory of thalamic function. J Comp Neurol 454:482-494.

Watanabe K, Kawana E (1980) A horseradish peroxidase study on the mammillothalamic tract in the rat. Acta Anat (Basel) 108:394-401.

Wolff M, Loukavenko EA, Will BE, Dalrymple-Alford JC (2008) The extended hippocampal-diencephalic memory system: enriched housing promotes recovery of the flexible use of spatial representations after anterior thalamic lesions. Hippocampus 18:996-1007. 